\title{
Thermal effects of interface motion in crystallization
}

\author{
A Umantsev \\ Department of Chemistry/Physics, Fayetteville State University, Fayetteville, NC 28301, USA
}

Received 29 August 2011, in final form 10 April 2012

Published 2 May 2012

Online at stacks.iop.org/MSMSE/20/045013

\begin{abstract}
This paper discusses two thermal effects of crystallization, which may be of interest for the community of molecular dynamics modelers. The first effect deals with the problem of motion of a plane interface in the system with internal cooling. It provides a simple recipe for identification of the kinetic coefficient of growth as a function of the measurable quantities, which does not require direct measurement of the interfacial temperature during the crystallization. The second effect deals with a heat-trapping effect, which consists in the crystallization of a solid phase from the supercooled liquid when the temperature of the crystallized solid is above the melting point.
\end{abstract}

(Some figures may appear in colour only in the online journal)

\section{Introduction}

Over the years molecular dynamics (MD) has become a powerful tool in the study of the most important phase transformation-crystallization. Finally, the modelers achieved the state of the art of the simulations, which allows them to see and analyze the thermal effects of the crystal-melt interface motion [1], the effects that the theorists have been studying for decades [2,3]. Recently, Monk et al [4] included the effects of generation and dissipation of latent heat in the procedure of identification of interfacial mobility in the MD crystallization of $\mathrm{Ni}$ and obtained a significant deviation from the isothermal result. Although presenting a significant step forward in the direction of identification of the important kinetic parameters of crystallization of simple metals, this study has a few problems that may be removed in order to achieve the highest accuracy of measurements. The main error of the kinetic coefficient measurement comes from the measurement of the interfacial temperature. Hence, it would be beneficial to find a method of determining the kinetic coefficient that does not require direct measurement of the interfacial temperature in MD crystallization. In section 2 we present an exact solution of the problem of motion of a plane interface in the system with internal cooling mechanism. Significance of the solution is that, on the one hand, it represents a situation that can be easily replicated by the MD method and, on the other hand, it provides a simple formula for the interfacial mobility as a function of the measurable quantities.

Section 3 is devoted to a heat-trapping effect, which is one of the most intriguing thermal effects of interfacial motion. This effect consists in the crystallization of a solid phase from 
the supercooled liquid when the temperature of the crystallized solid is above the equilibrium point. Heat trapping is a combination of the effects of low temperature gradient at the interface, large interfacial mobility, large heat of transformation and broad interface. This effect was theoretically predicted by Roitburd and the author [5,6] and then independently rediscovered by Patashinsky and Chertkov [7] and Schofield and Oxtoby [8]. However, to describe the effect, all the authors used the same field-theoretic method. General scientific validation of the effect depends on its verification by at least one more, independent method. Experimental verification of the effect is hampered by the material-parameters criterion outlined above, which is not fulfilled for any of the substances known to the author. The author believes that the method of MD simulations is the most appropriate one for the purpose of verification of the effect and that heat trapping can be detected by the MD crystallization of simple metals.

\section{Kinetic coefficient}

A reliable method of identification of the kinetic coefficient of growth should be based on a physical regime where the crystal-melt interface moves with a constant speed, at least asymptotically, because otherwise it is very difficult to discriminate between the asymptotic and transient in a non-stationary regime. As known $[9,10]$, a stationary regime does exist for the crystallization problem controlled by the kinetics of attachment of atoms at the interface:

$$
v=\mu\left(T_{\mathrm{M}}-T_{\mathrm{I}}\right) \text {. }
$$

Here $T_{\mathrm{M}}$ is the melting point, $T_{\mathrm{I}}$ is the temperature, $v$ is the speed of the crystal $/ \mathrm{melt}$ interface and $\mu$ is the sought kinetic coefficient. Unfortunately, this regime requires a very high level of supercooling ( $>500 \mathrm{~K}$ for $\mathrm{Ni}$ ), which may not be attainable in the MD simulations.

Fortunately, the problem of crystallization under the conditions of internal cooling has a stationary regime even for small undercoolings of the melt. The one-dimensional (1D) temperature field of such a system $T(x, t)$ changes due to the process of thermal conduction:

$$
C\left(\frac{\partial T}{\partial t}\right)_{\text {cond }}=\lambda \frac{\partial^{2} T}{\partial x^{2}}
$$

and due to the heat sinks, which are uniformly distributed over the whole system:

$$
C\left(\frac{\partial T}{\partial t}\right)_{\text {sink }}=-h\left(T-T_{\mathrm{L}}\right) .
$$

In equation (2) $\lambda$ is the thermal conductivity and $C$ is the specific heat, considered to be equal in both phases. In equation (3) $T_{\mathrm{L}}$ is the temperature of the surrounding environment, which is below the melting point $T_{\mathrm{M}}$. Due to release of the latent heat of crystallization $L$ at the interface its temperature is $T_{\mathrm{I}}>T_{\mathrm{L}}$. In equation (3) $h$ is the internal heating/cooling coefficient, which yields the internal heating/cooling relaxation time:

$$
\tau=\frac{C}{h} \text {. }
$$

In the MD simulations the internal heating/cooling may be realized with the help of the NoséHoover thermostats [11, 12].

Combining the process of conductivity, equation (2), and internal cooling, equation (3), we arrive at the $1 \mathrm{D}$ heat equation of the form

$$
C \frac{\partial T}{\partial t}=\lambda \frac{\partial^{2} T}{\partial x^{2}}-h\left(T-T_{\mathrm{L}}\right)
$$

Details of the formulation of the problem of crystallization of the melt with the initial temperature $T_{\mathrm{L}}$ and its rigorous solution using the sharp-interface approximation are provided 
in the appendix. The general solution of the problem provided by equations (A13) and (A14) may be resolved for the kinetic coefficient:

$$
\frac{1}{\mu}=\frac{T_{\mathrm{M}}-T_{\mathrm{L}}}{v}-\frac{L}{\sqrt{4 \lambda h+C^{2} v^{2}}} .
$$

Formula (6) allows one to calculate the kinetic coefficient $\mu$ if the interfacial velocity $v$ and the bulk properties of the system, such as the internal heating/cooling coefficient, are known. Instead of the temperature of the interface, formula (6) requires knowledge of the base temperature $T_{\mathrm{L}}$. Formula (6) also shows that the process described by equations (1)-(6) is characterized by the critical thermostat-relaxation time:

$$
\tau_{\mathrm{c}} \equiv \frac{4 \lambda}{C v^{2}} \approx 10 \mathrm{~ns}
$$

such that for sluggish thermostats ( $\tau \approx \tau_{\mathrm{c}}$, case 1 of the appendix) the internal heating/cooling is not essential and the process is essentially adiabatic (the parameters for equation $(6 a)$ were taken from [4]).

Rigorously speaking, solution (A13), (A14) and formula (6) are valid for an infinitely long system only. In a finite-size system the stationary regime may be achieved asymptotically by increasing the size of the system.

\section{Heat trapping in MD crystallization}

In this section we consider another thermal effect of interface motion-heat trapping. As noted in section 1, this effect consists in the crystallization of a solid phase from the supercooled liquid when the temperature of the crystallized solid is above the equilibrium point. In distinction to the regime considered in the previous section, heat trapping does not need internal cooling; most clearly this effect is revealed under the conditions of an adiabatic system. Another difference of the heat-trapping regime from the one of the previous section is that the thickness of the moving interface $l$ is a decisive factor for the effect, so that if the interface is thinner than the critical value the effect does not take place. Namely, the criterion for heat trapping to be possible $[5,6]$ is

$$
\mathrm{Ht} \equiv \frac{\lambda}{\mu L l}<\frac{1}{6}
$$

where $\mathrm{Ht}$ is the heat-trapping number. For MD-Ni [4], unfortunately, $\mathrm{Ht}=1.1$ that is, the criterion is not fulfilled.

Let us consider the heat-trapping effect in detail. For a stationary regime under adiabatic conditions:

$$
T(x \rightarrow \infty) \equiv T_{\mathrm{S}}=T_{\mathrm{L}}+\frac{L}{C}=T_{\mathrm{M}}-\frac{L}{C}(\Delta-1)
$$

which is nothing more than an expression of the conservation of energy condition. Hence, equation (8) is valid regardless of the internal structure of the interface. As one can see from section 2 and the appendix, for a sharp interface, the stationary regime of growth exists for the supercoolings $\Delta>1[9,10]$. In this case, $v=\mu L(\Delta-1) / C$, see equation (A17), and the temperature of the growing crystal is $T_{\mathrm{S}}<T_{\mathrm{M}}$, see equation (8). For $0<\Delta<1$ the stationary regime is replaced by a self-similar regime with time-decaying velocity $[2,10]$, but the relation $T_{\mathrm{S}}<T_{\mathrm{M}}$ is preserved. Equations (8) and (A17) are depicted in figure 1 in blue.

A theoretical analysis [6] shows that if the thickness of the interface is taken into account then the solution, equation (A17), is replaced by the following:

$$
\Delta=1+(\mathrm{Ht}-0.1583) P e+0.0403 P e^{2}-O\left(P e^{3}\right)
$$




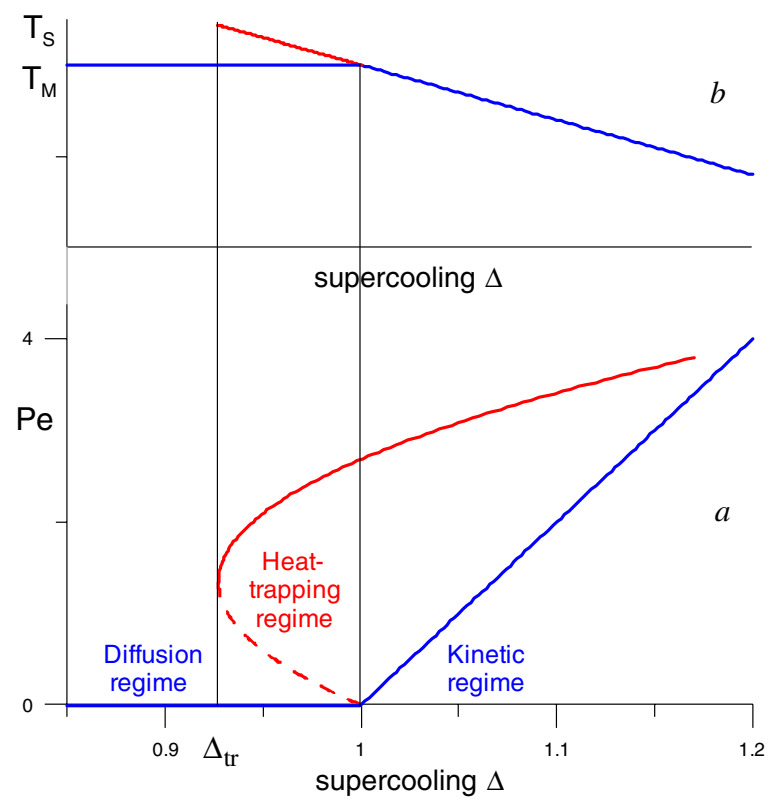

Figure 1. Different regimes of motion of a plane interface separating solid and liquid phases: (a) Peclet number $P e$ and $(b)$ final temperature of the solid phase $T_{\mathrm{S}}$ versus the initial supercooling $\Delta$ of the liquid phase. Blue lines-solution of the Stefan problem and equation (8), (A17); red lines-heat-trapping solution, equations (8) and (9), for $\mathrm{Ht}=0.05$.

where $P e$ is the Peclet number of the process:

$$
P e \equiv \frac{C l v}{\lambda} \text {. }
$$

If the criterion, equation (7), is not fulfilled then the stationary regime $(v>0)$ exists for $\Delta>1$ only and the difference from the sharp-interface case is insignificant. If the criterion, equation (7), is fulfilled then the stationary regime $(v>0)$ exists also for

$$
1-\frac{(\mathrm{Ht}-0.1583)^{2}}{4 \times 0.0403} \equiv \Delta_{\mathrm{tr}} \leqslant \Delta<1
$$

One may say that in this case the kinetic regime of growth 'penetrates' the temperature domain of the diffusion regime. In figure 1 the function $\operatorname{Pe}(\Delta)$, equation (9), is depicted in red for the case when criterion (7) is fulfilled. However, the most interesting part of the effect is revealed if, using equations (8)-(10), we calculate the final temperature of the solid phase after the transformation. One can see (red line in figure $1(b)$ ) that this temperature is above the equilibrium point $T_{\mathrm{S}}>T_{\mathrm{M}}$. During this process the solid phase grows $(v>0)$ at a temperature above the equilibrium point $\left(T_{\mathrm{S}}>T_{\mathrm{M}}\right)$.

So far we have considered a stationary process, that is the process in an infinitely long system. When the crystal/melt interface approaches the boundary of a finite-size system there are two possible scenarios of the heat-trapping effect: (1) the interface bounces off the boundary and remelts a portion of the crystallized material; (2) the interface moves all the way to the end and the whole sample crystallizes throughout. In the first scenario the system finds the state of global equilibrium - the two-phase state of coexistence at the temperature of the melting point. In the second scenario the system overshoots the global equilibrium and ends up in the metastable state of the homogeneous crystal phase at a temperature above the melting point. 


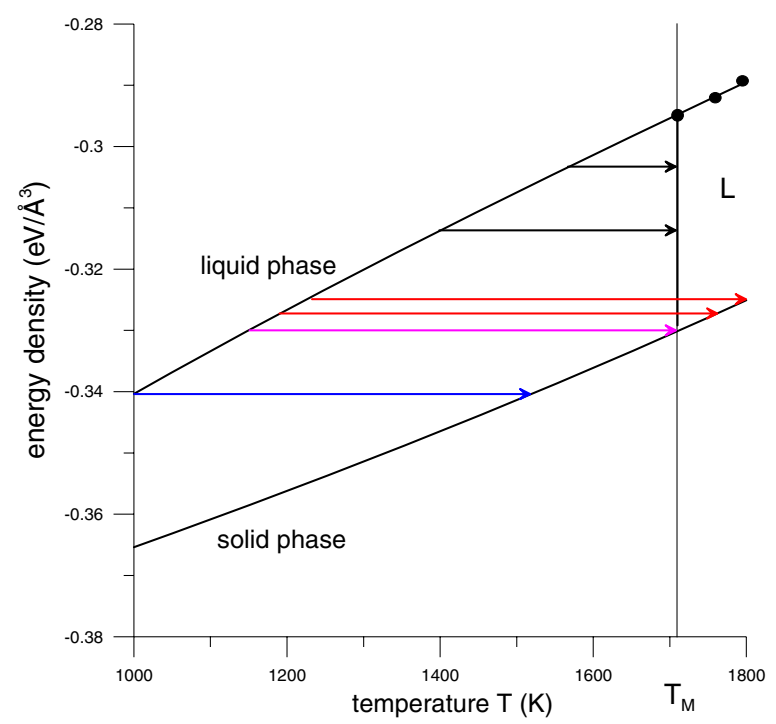

Figure 2. Phase-transformation scenarios in a finite-size system of MD-Ni [4]. Black dots-no crystallization; blue arrow-adiabatic crystallization; black arrows-adiabatic transformation into the globally stable two-phase state; red arrows - the heat-trapping regime.

Both scenarios were observed in the simulations of adiabatic phase transformations using the field-theoretic method (see figure 12 and the corresponding comments in [13]). Various outcomes of the phase-transformation scenarios in the finite-size system are expressed in the diagram of the internal energy density versus temperature, figure 2 . If the initial temperature of the liquid phase $T_{\mathrm{L}}$ is above the melting point $T_{\mathrm{M}}$, the crystallization does not occur at all (black dots). If $T_{\mathrm{L}}<T_{\mathrm{M}}-L / C$, the final outcome of the transformation is a homogeneous solid phase at a temperature below the melting point-adiabatic crystallization (blue arrow). If $T_{\mathrm{M}}-L / C<T_{\mathrm{L}}<T_{\mathrm{M}}$, then the global equilibrium is achieved at the two-phase state of variable phase fraction and temperature equal to the melting point $T_{\mathrm{M}}$ (black arrows). However, if criterion (7) is fulfilled then the regime of crystallization is possible when the system overshoots the global equilibrium and ends up in a homogeneous solid state at a temperature above the melting point. The heat-trapping regime is possible in a limited range of initial temperature of the liquid phase $T_{\mathrm{M}}-L / C<T_{\mathrm{L}}<T_{\mathrm{tr}}=T_{\mathrm{M}}-L D_{\mathrm{tr}} / C$ (red arrows).

\section{Acknowledgments}

The author would like to thank Dr J Hoyt for helpful discussions on the paper. This work was supported by the ARO Grant W911NF-06-1-0302.

\section{Appendix A. Crystallization with internal cooling}

\section{A.1. Formulation}

We consider crystallization in an infinitely long thin rod immersed into a medium of temperature $T_{\mathrm{L}}$ below the melting point $T_{\mathrm{M}}$. The medium provides uniformly distributed internal cooling to the rod and the temperature distribution in the rod is described by equation (5). Initially the liquid is cooled to the same temperature $T_{\mathrm{L}}$, which determines the far-field boundary 
conditions:

$$
T \rightarrow T_{\mathrm{L}} \quad \text { if } x \rightarrow \pm \infty .
$$

We disregard any deviations of the propagating front of crystallization from planar geometry (morphological stability is not broken). The speed of the front $v$ is determined by the thermal characteristics of the system as well as by the kinetic coefficient $\mu$, equation (1). The heat released at the front is removed by heat conduction; this process is described by the Stefan heat-balance interface condition:

$$
\left.\lambda \frac{\partial T}{\partial x}\right|_{x_{\mathrm{I}}-0}-\left.\lambda \frac{\partial T}{\partial x}\right|_{x_{\mathrm{I}}+0}=L v
$$

where $x_{\mathrm{I}}$ is the coordinate of the interface.

\section{A.2. Solution}

We seek a stationary (travelling wave) solution of the type

$$
T(x, t)=T_{\mathrm{L}}+\frac{L}{C} \theta(u), \quad u=\sqrt{\frac{h}{\lambda}}(x-v t) .
$$

Then the problem, equations (1), (5), (A1)-(A3), takes the form of an ODE:

$$
\theta^{\prime \prime}+p \theta^{\prime}-\theta=0, \quad-\infty<u<+\infty
$$

with the conditions on the stationary boundary (interface):

$$
\begin{array}{ll}
\theta^{\prime}(0-0)-\theta^{\prime}(0+0)=p & \text { at } u=u_{\mathrm{I}}=0 \\
v=\mu \frac{L}{C}[\Delta-\theta(0 \pm 0)] & \text { at } u=u_{\mathrm{I}}=0
\end{array}
$$

and the boundaries of the system far away from the interface:

$$
\theta(u) \rightarrow 0 \quad \text { at } u \rightarrow \pm \infty .
$$

In equation (A6)

$$
\Delta \equiv \frac{C\left(T_{\mathrm{M}}-T_{\mathrm{L}}\right)}{L}>0
$$

is the dimensionless supercooling $\left(T_{\mathrm{M}}-T_{\mathrm{L}}\right)$.

The general solution of the problem, equations (A4)-(A6), is

$$
\theta=A_{+} \mathrm{e}^{\omega_{+} u}+A_{-} \mathrm{e}^{\omega_{-} u}, \quad \omega_{ \pm}=-\frac{p}{2} \pm \sqrt{1+\frac{p^{2}}{4}} .
$$

To satisfy the far-field boundary condition, equation (A7), we must choose

$$
\theta= \begin{cases}A_{+} \mathrm{e}^{\omega_{+} u}, & -\infty<u \leqslant 0 \\ A_{-} \mathrm{e}^{\omega_{-} u}, & 0 \leqslant u<+\infty\end{cases}
$$

Satisfying the kinetic interface condition, equation (A6), we obtain

$$
A_{+}=A_{-}=\Delta-n p, \quad n \equiv \frac{\sqrt{\lambda h}}{\mu L} .
$$

Satisfying the heat-balance condition, equation (A5), we obtain

$$
A=\frac{p}{\sqrt{4+p^{2}}} \text {. }
$$

6 


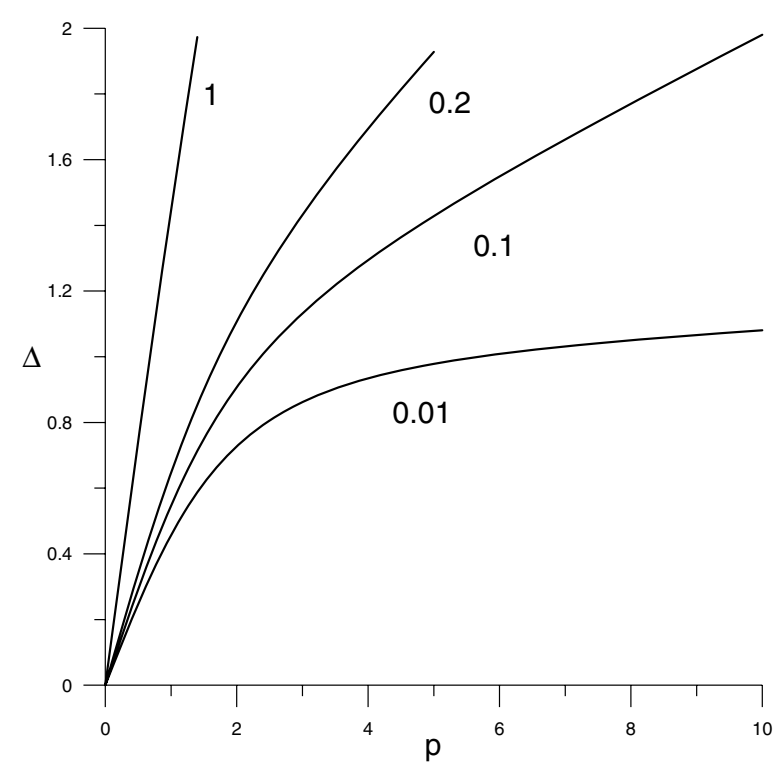

Figure A1. Graphical solution of equation (A13) for various values of the parameter $n$, which label the curves.

Thus, equations (A11) and (A12) yield an equation for $p$ :

$$
\Delta=n p+\frac{p}{\sqrt{4+p^{2}}},
$$

which must be satisfied in order for the solution, equations (A9) and (A10), to exist. For the speed of the interface motion equations (A6) and (A13) yield

$$
v=\mu \frac{L}{C} n p=\frac{\sqrt{\lambda h}}{C} p
$$

The solution, equations (A9), (A10), (A13) and (A14), has two limiting cases.

Case 1.

$$
\Delta>1, \quad n \ll \Delta-1 .
$$

In this case,

$$
p \approx \frac{\Delta-1}{n} \gg 1, \quad \omega_{+} \approx \frac{1}{p}, \quad \omega_{-} \approx-p, \quad A \approx 1 ;
$$

the speed of the interface is

$$
v \approx \mu \frac{L}{C}(\Delta-1)
$$

and the temperature distribution is

$$
T(x, t) \approx \begin{cases}T_{\mathrm{L}}+\frac{L}{C} \mathrm{e}^{\frac{h}{\mu L(\Delta-1)}(x-v t)}, & x<v t \\ T_{\mathrm{L}}+\frac{L}{C} \mathrm{e}^{-\frac{\mu L}{\lambda}(\Delta-1)(x-v t)}, & x>v t .\end{cases}
$$

In this case internal cooling is not essential. 


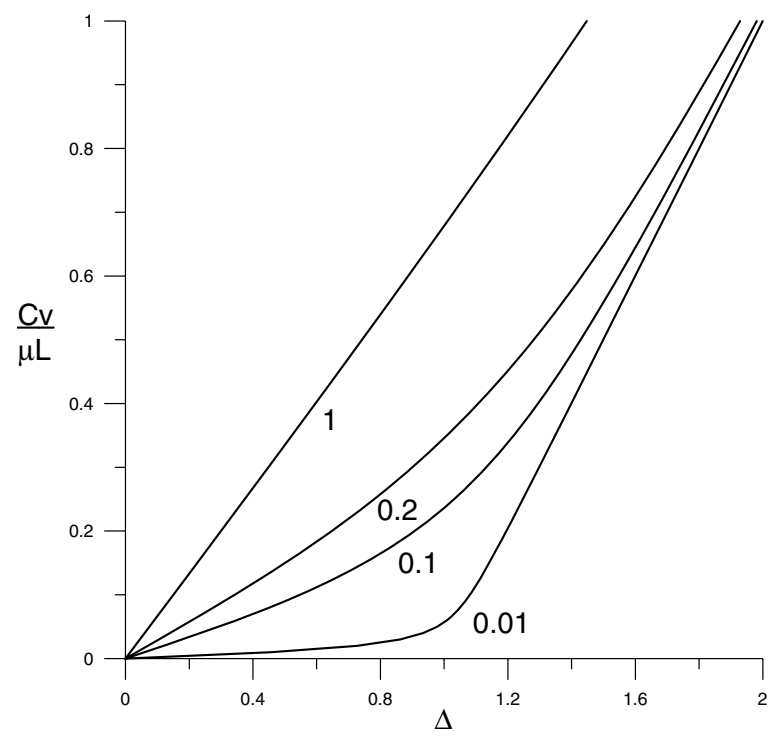

Figure A2. Speed of the interface motion equation (A14) for various values of the parameter $n$, which label the curves.

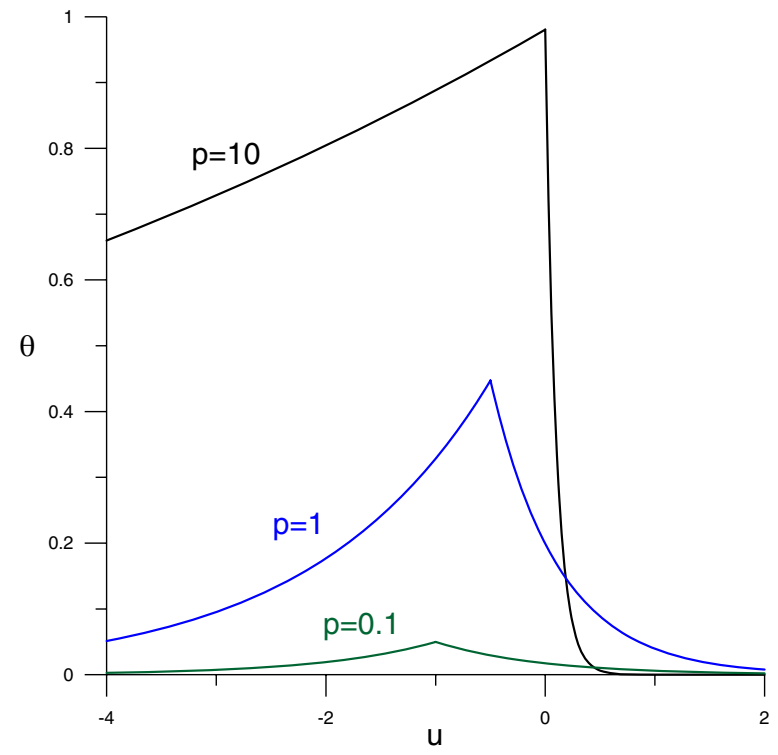

Figure A3. Temperature distribution in the system, equation (A10), for various values of $p$, which label the curves. The coordinates of the fronts are the cusps of the distributions, which are arbitrarily shifted with respect to each other.

Case 2.

$$
\Delta \ll 1 \quad \text { or } \quad n \gg \Delta \sim 1
$$

In this case,

$$
p=\frac{2 \Delta}{1+2 n} \ll 1, \quad \omega_{ \pm} \approx \pm 1, \quad A \approx \frac{\Delta}{1+2 n} \ll 1
$$


the speed of the interface is

$$
v=\mu\left(T_{\mathrm{M}}-T_{\mathrm{L}}\right) \frac{2 n}{1+2 n}
$$

and the temperature distribution is

$$
T(x, t) \approx \begin{cases}T_{\mathrm{L}}+\frac{T_{\mathrm{M}}-T_{\mathrm{L}}}{1+2 n} \mathrm{e}^{+\sqrt{\frac{h}{\lambda}}(x-v t)}, & x<v t \\ T_{\mathrm{L}}+\frac{T_{\mathrm{M}}-T_{\mathrm{L}}}{1+2 n} \mathrm{e}^{-\sqrt{\frac{h}{\lambda}}(x-v t)}, & x>v t .\end{cases}
$$

In this case internal cooling is essential.

In figures A1, A2 and A 3 the graphical solution of equation (A13), the speed of the interface motion, equation (A14), and the temperature distribution in the system, equation (A10), are depicted respectively. Cases 1 and 2 can be identified by the respective parameters.

\section{References}

[1] Mendelev M I, Rahman M J, Hoyt J J and Asta M 2010 Modeling Simul. Mater. Sci. Eng. 18074002

[2] Rubinstein L I 1971 The Stefan Problem (Providence, RI: American Mathematical Society)

[3] Umantsev A 2007 Thermal effects in phase transformations: a review Physica D 2351

[4] Monk J, Yang Y, Mendelev M I, Asta M, Hoyt J J and Sun D Y 2010 Modelling Simul. Mater. Sci. Eng. 18015004

[5] Umantsev A and Roitburd A 1988 Sov. Phys._Solid State 30 651-5

[6] Umantsev A 1992 J. Chem. Phys. 96 605-17

[7] Patashinsky A Z and Chertkov M V 1990 Fiz. Tverd. Tela (Leningrad) 32509

Patashinsky A Z and Chertkov M V 1990 Sov. Phys._Solid State 32295

[8] Schofield S A and Oxtoby D W 1991 J. Chem. Phys. 942176

[9] Rosenthal J 1946 Trans. ASME 68849

[10] Umantsev A 1985 Sov. Phys._Crystallogr. 30 87-91

[11] Nosé S 1984 J. Chem. Phys. 81511

[12] Hoover W G 1985 Phys. Rev. A 311695

[13] Umantsev A and Olson G B 1993 Phys. Rev. E 484229 